\title{
Orbital currents and cheap vortices in underdoped cuprates
}

\author{
Patrick A. Lee \\ Center for Materials Science and Engineering and Department of Physics, MIT, Cambridge, MA 02139 USA
}

(November 9, 2018)

\begin{abstract}
In the past several years, we have developed a theory for the underdoped cuprates in collaboration with X.-G. Wen, based on an $\mathrm{SU}(2)$ formulation of the $t-J$ model. In this formulation, the staggered flux state plays a central role as the progenitor of the Néel state at half-filling and a close competitor to the $d$-wave superconductor with small doping where it is characterized by staggered orbital currents. We found support for this point of view when we discovered fluctuating orbital currents in the Gutzwiller projected BCS wavefunction. We shall argue that low-energy vortices, where the staggered flux state is stabilized in the core, are needed to explain many of the unusual properties of underdoped cuprates. Proposed experiments to look for these orbital currents will be discussed.
\end{abstract}

The phenomenon of high temperature superconductivity in cuprates is generally agreed to be associated with doping into a Mott insulator. The undoped material is an antiferromagnet with a large exchange energy $J$ of order $1500 \mathrm{~K}$. The doped holes hop with a matrix element $t$, which is estimated to be approximately $3 J$. However, the Néel state is not favorable for hole hopping, because after one hop the spin finds itself in a ferromagnetic environment. Thus it is clear that the physics is that of competition between the exchange energy $J$ and the hole kinetic energy per hole $x t$. Apparently the superconducting state emerges as the best compromise, but how and why this occurs is the central question of the high $\mathrm{T}_{c}$ puzzle. In the underdoped region this competition results in physical properties that are most anomalous. The metallic state above the superconducting $\mathrm{T}_{c}$ behaves in a way unlike anything we have encountered before. Essentially, an energy gap appears in some properties and not others, and this metallic state has been referred to as the pseudogap state. We will focus our attention on this region because the phenomenology is well established and we have the best chance of sorting out the fundamental physics. Indeed, I will argue that a consensus is emerging on the framework needed to describe this phenomenon and that detailed proposals are amenable to experimental tests.

The pseudogap phenomenon is most clearly seen in the uniform susceptibility. For example, Knight shift measurement in the YBCO 124 compound shows that while the spin susceptibility is almost temperature independent between $700 \mathrm{~K}$ and $300 \mathrm{~K}$, as in an ordinary metal, it decreases below $300 \mathrm{~K}$ and by the time the $\mathrm{T}_{c}$ of $80 \mathrm{~K}$ is reached, the system has lost $80 \%$ of the spin susceptibility. 1 Similarly, the linear $T$ coefficient of the specific heat shows a marked decrease below room temperature. It is apparent that the spins are forming into singlets and the spin entropy is gradually lost. On the other hand, the frequency dependent conductivity behaves very differently depending on whether the electric field is in the $a b$ plane $\left(\sigma_{a b}\right)$ or perpendicular to it $\left(\sigma_{c}\right)$. At low frequencies (below $\left.500 \mathrm{~cm}^{-1}\right)\left(\sigma_{a b}\right)$ shows a typical Drude-like behavior for a metal with a width which decreases with temperature, but an area (spectral weight) which is independent of temperature 2 Thus there is no sign of the pseudogap in the spectral weight. This is surprising because in other examples where an energy gap appears in a metal, such as the onset of charge or spin density waves, there is a redistribution of the spectral weight from the Drude part to higher frequencies. On the other hand below $300 \mathrm{~K} \sigma_{c}(\omega)$ is gradually reduced below $500 \mathrm{~cm}^{-1}$ and a deep hole is carved out of $\sigma_{c}(\omega)$ by the time $\mathrm{T}_{c}$ is reached. Finally, angle-resolved photoemission shows that an energy gap (in the form of a pulling back of the leading edge of the electronic spectrum from the Fermi energy) is observed near momentum $(0, \pi)$ and the onset of superconductivity is marked by the appearance of a small coherent peak at this gap edge.

The pseudogap phenomenology is well explained by a cartoon picture which emerges from the RVB (resonating valence band) theory of Anderson. 3 The spins are paired into singlet pairs. However, the pairs are not static but are fluctuating due to quantum mechanical superposition, hence the term quantum spin liquid. The singlet formation explains the appearance of the spin gap and the reduction of spin entropy. The doped holes appear as vacancies in the background of singlet pair liquid and can carry a current without any energy gap. However in $c$-axis conductivity and electron is removed from one plane and placed on the next. The intermediate state is an electron which carries spin $1 / 2$ and therefore it is necessary to break a singlet pair and pay the spin-gap energy. The same consideration applies to the photoemission experiment. Finally, according to RVB theory, superconductivity emerges when the holes become phase coherent. The spin singlet familiar in the BCS theory has already been formed.

While the above picture is appealing, there has been another popular explanation which at first sight sounds less exotic. The idea is that the pseudogap phenomenology can be understood as a superconductor with robust amplitude but strong phase fluctuation. The superfluid density $\rho_{s}$ which controls the phase stiffness is proportional to the doping concentration $x$ and becomes small in the underdoped region. As emphasized by Uemurat and by Emery and Kivelson, $5 \mathrm{~T}_{c}$ is controlled by $\rho_{s}$ and is much lower than the energy gap. We shall now argue that these two points of views are not unrelated to each other. Setting aside the question of where the strong pairing amplitude comes from in the first place, that the phase fluctuation scenairo is incomplete can be seen from the following argument. In 
two dimensions the destruction of superconducting order is via the Berezinskii-Kosterlitz-Thouless (BKT) theory of vortex unbinding. Above $\mathrm{T}_{c}$ the number of vortices proliferate and the normal metallic state is reached only when the vortex density is so high that the cores overlap. (There is considerable lattitude in specifying the core radius, but this does not affect the conclusion.) At lower vortex density, transport properties will resemble a superconductor in the flux flow regime. In ordinary superconductors, the BKT temperature is close to the mean field temperature, and the core energy rapidly becomes small. However, in the present case, it is postulated that the mean field temperature is high, so that a large core energy is expected. Indeed, in a conventional core the order parameter and energy gap vanish, costing $\Delta_{0}^{2} / E_{F}$ per unit area of energy. Using a core radius of $\xi=V_{F} / \Delta_{0}$, the core energy of a conventional superconductor is $E_{F}$. In our case, we may replace $E_{F}$ by $J$. If this were the case, the proliferation of vortices would not happen until a high temperature $\sim J$ independent of $x$ is reached. Thus for the phase fluctuation scenario to to work, it is essential to have "cheap" vortices, with energy cost of order $\mathrm{T}_{c}$. Then the essential problem is to understand what the vortex core is made of. Put another way, there has to be a competing state with energy very close to the $d$-wave superconductors which constitute the core. The vortex core indeed offers a glimpse of the normal state reached when $H$ exceeds $H_{c 2}$, and is an important constituent of the pseudogap state above $\mathrm{T}_{c}$.

To summarize, the pseudogap phenomenology is so clearly defined by experiments that I believe a broad consensus on the theoretical framework is emerging. The following points are common to a number of investigators.

1. The key issue is how holes are accommodated in a doped Mott insulator.

2. The superconducting state is characterized by a large energy gap and a small $\rho_{s}$. The transition temperature is controlled by phase coherence.

3. The superconducting state does not evolve out of a normal state with well defined quasiparticles. Therefore the question of pairing mechanisms in the traditional sense of identifying the boson being exchanged between quasiparticles is not the right question.

4. What is needed is to identify the competing state which lives iside the vortex core.

Whatare the candidates for the competing order? A candidate which has attracted a lot of attention is the stripe phase.6. In the LSCO familty, dynamical stripes (spin density waves) are clearly important, especially near $x=\frac{1}{8}$. There are recent report of incommensurate SDW nucleating around vortices. However, until now there has been little evidence for stripes outside of the LSCO family. On the theoretical side, as a competing state it is not clear how the stripes are connected to $d$-wave superconductivity and it is hard to understand how the nodal quasiparticles turn out to be most sharply defined on the Fermi surface, since these have to transverse the stripes at a $45^{\circ}$ angle.

A second candidate for the vortex core is the antiferromagnetic state. This possibility was first proposed several years ago in the context of the $\mathrm{SO}(5)$ theory $\mathrm{B}$ This theory is phenomenological in that it involves only bosonic degrees of freedom (the SDW and pairing order parameters). The quasiparticles are out of the picture and indeed this theory applies equally well to $d$-wave or a fully gapped $s$-wave superconductor. Thus the fundamental question of how the holes are accommodated has not really been addressed. The nucleation of SDW around the core in the LSCO family lends support to the existence of antiferrmagnetic order inside the core. Furthermore, there are reports of enhanced antiferromagnetic spin fluctuations, and perhaps even static order, using NMR.9.10 I shall argue next that other considerations also lead to antiferromagnetic fluctuations and possibly static orders inside the vortex core, so that the observation of antiferromagnetic cores does not necessarily imply the existence of $\mathrm{SO}(5)$ symmetry.

Finally, I come to the candidate which we favor - the staggered flux state with orbital currents.11 Indeed, Lee and Wen have successfully constructed a "cheap" vortex state.12 The staggered flux phase has an advantage over other possibilities in that its excitation spectrum is similar to the $d$-wave superconductor and the $\mathrm{SU}(2)$ theory allows us to smoothly connect it to the superconductivity. We also regard the staggered flux phase as the precursor to Néel order, so that antiferromagnetic fluctuations or even SDW order are accommodated naturally. Of course, it is experiments which have the final say as to which candidate turns out to be realized. Our strategy is to work out as many experimental consequences as we can and propose experiments to confirm or falsify gur theory.

The staggered flux state was first introduced as a mean field solution at half-filling 13 and later was extended to include finite doping.14 At half-filliing, due to the constraint of no double occupation, the staggered flux state corresponds to an insulating state with power law decay in the spin correlation function. It is known that upon including gauge fluctuations which enforce the constraint, the phenomenon of confinement and chiral symmetry breaking occurs, which directly corresponds to Néel ordering. 15 The idea is that with doping, confinement is suppressed at some intermediate energy scale, and the state can be understood as fluctuating between the staggered flux state and the $d$-wave superconducting state. Finally, when the holes become phase coherent, the $d$-wave superconducting state is the stable ground state. Thus the staggered flux state may be regarded as the "mother state" which is an unstable fixed point due to gauge fluctuations. It flows to Néel ordering at half-filling and to the $d$-wave superconductor for 
sufficiently large $x$. Thus the staggered flux state plays a central role in this kind of theory. We should point out that the staggered flumstate (called the $D$-density wave state) has recently been proposed as the ordered state in the pseudogap region. 1 As explained elsewhere 17 we think that this view is not supported by experiment and we continue to favor the fluctuation picture.

The above picture finds support from studies of projected wavefunctions, where the no-double-occupation constraint is enforced by hand on a computer. With doping the best state is a projected $d$-wave state. Recently we calculated the current-current correlation function of this state $c_{j}(k, \ell)=<j(k) j(\ell)>$ where $j(k)$ is the physical electron current on the bond $k$. The average current $\langle j(k)\rangle$ is obviously zero, but the correlator exhibits a staggered circulating pattern.18 Such a pattern is absent in the $d$-wave BCS state before projection, and is a result of the Gutzwiller projection. Our result for $c_{j}$ is consistent with exact diagonalization of two holes in 32 sites.19

The staggered current generates a staggered physical magnetic field (estimated to be 10-40 gauss) 14.18 which may be detected, in principle, by neutron scattering. In practice the small signal makes this a difficult, though not impossible experiment and we are motivated to look for situations where the orbital current may become static or quasi-static. Recently, we analyzed the structure of the $h c / 2 e$ vortex in the superconducting state within the SU(2) theory and concluded that in the vicinity of the vortex core, the orbital current becomes quasi-static, with a time scale determined by the tunnelling between two degenerate staggered flux states. 12 It is very likely that this time is long on the neutron time scale. Thus we propose that a quasi-static peak centered around $(\pi, \pi)$ will appear in neutron scattering in a magnetic field, with intensity proportional to the number of vortices. The time scale may actually be long enough for the small magnetic fields generated by the orbital currents to be detectable by $\mu$-SR or Yttrium NMR. Again, the signal should be proportional to the external fields. (The NMR experiment must be carried out in 2-4-7 or 3 layer samples to avoid the cancellation between bi-layers.) We have also computed the tunnelling density of states in the vicinity of the vorte core, and predicted rather specific kind of period doubling which should be detectable by atomic resolution STM 20 The recent report 21 of a static field of \pm 18 gauss in underdoped YBCO which appears in the vortex state is promising, even though muon cannot distinguish between orbital current or spin as the origin of the magnetic field. We remark that in the underdoped antiferromagnet, the local moment gives rise to a field of 340 gauss at the muon site. Thus if the 18 gauss signal is due to spin, it will correspond to roughly $1 / 20$ th of the full moment.

We remark that our analytic model of the vortex core is in full agreement with the numerical solution of unrestricted mean field $\Delta_{i j}$ and $\chi_{i j}$ by Wang, Han and Lee.22 Recently we feund that for small doping in the $t$ - $J$ model a small moment SDW cexists with orbital currents in the vortex core.23 More generally, we expect $(\pi, \pi)$ spin fluctuations to be enhanced 24 so that the tendency to antiferromagnetism is fully compatible with the staggered flux picture. This vortex solution is also interesting in that the tunnelling density of states show a gap, with no sign of the large resonance associated with Caroli-deGennes-type core levels found in the standard BCS model of the vortex. There exists a single bound state at low lying energy, 3 in agreement with STM experiments. The low density of states inside the vortex core has an important implication. In the standard Bardeen-Stephen model of flux-flow resistivity, the friction coefficient of a moving vortex is due to dissipation associated with the vortex core states. Now that the core states are absent, we can expect anomalously small friction coefficients for underdoped cuprates. The vortex moves fast transverse to the current and gives rise to large flux-flow resistivity. Since the total conductivity is the sum of the flux-flow conductivity and the quasiparticle conductivity, it is possible to get into a situation where the quasiparticle conductivity dominates even for $\mathrm{H} \ll \mathrm{H}_{c 2}$. Thus the "cheap" and "fast" vortex opens the possibility of having vortex states above the nominal $\mathrm{T}_{c}$ and $\mathrm{H}_{c 2}$, when the resistivity looks like that of a metal, with little sign of flux-flow contribution. From this point of view, the large Nerst effect observed by Ong and co-worker\$25 over a large region in the $\mathrm{H}-\mathrm{T}$ plane above the nominal $\mathrm{T}_{c}$ and $\mathrm{H}_{c 2}$ (as determined by resistivity) may be qualitatively explained.

Finally, I would like to mention my recent work with N. Nagaosa on new collective modes in the superconductor state.26 In ordinary superconductors, there is a single pairing order parameter $\Delta$ and associated with it are the amplitude mode and the phase modes. In our theory the hopping matrix element $\chi_{i j}$ is also a dynamical degree of freedom. The basic reason is that the hole hopping amplitude depends sensitively on the spin configuration, as we explained earlier, so that $\chi_{i j}$ encodes the effect due to the spin degree of freedom. With the pairing and hopping amplitudes as dynamic degrees of freedom, it is natural that we can expect new collective modes. These can be evaluated in a direct way within mean field theory in analogy with the standard RPA treatment, but the SU(2) theory allows us to classify them. We find two new classes of collective modes. The first is called the $\theta$-mode, which describes the local fluctuation towards the staggered flux phase, and has the effect of generating the orbital current correlation described earlier. The second is called the $\phi$-gauge mode. This mode involves the relative oscillation of the amplitudes of $\chi_{i j}$ and $\Delta_{i j}$ in such a way that $\left|\chi_{i j}\right|^{2}+\left|\Delta_{i j}\right|^{2}$ is constant, and this oscillation is modulation in a staggered way. Thus this mode is most important near $(\pi, \pi)$, but it disperses throughout $k$ space in a way which we have computed. The frequency of the mode is estimated to be of order $J$ for $x=0.15$. This mode couples to Raman scattering in the standard way so that optical Raman scattering may observe the mode at $\mathbf{k}=0$. X-Ray Raman scattering should be able to map out the entire dispersion, but higher energy resolution than what is currently 
available will be needed. It turns out the LSCO offers a special opportunity to couple to this mode. In the LTO phase the copper oxygen bonds are buckled in a staggered way. A uniform motion of the oxygen along the $c$-axis leads to a staggered modulation of the hopping matrix element. Thus we predict that a new collective mode at $(\pi, \pi)$ will show up as a side band of the buckling mode in $c$-axis conductivity. Unfortunately, the relative spectral weight of one side band is estimated to be very small (of order $5 \times 10^{-3}$ ), making its detection a challenging one for experimentalists.

I thank X.-G. Wen, N. Nagaosa, D. Ivanov, J. Kishine and Y. Morita for their collaboration on the work reviewed here. I acknowledge the support of NSF through the MRSEC program grant no. DMR98-08941.

${ }^{1}$ N. Curro et al., Phys. Rev. B56 (1997) 877.

${ }^{2}$ A.F. Santander-Syro et al., Phys. Rev. Lett. 88 (2002) 097005.

${ }^{3}$ P.W. Anderson, Science 235 (1987) 1196.

${ }^{4}$ Y. Uemura et al., Phys. Rev. lett. 66 (1991) 2665.

${ }^{5}$ V.J. Emery, S. Kivelson, Nature 374 (1995) 434.

${ }^{6}$ V.J. Emery, S. Kivelson, J.M. Tranquada, Proc. Ntl. Acad. Sci. USA 96 (1999) 8814.

${ }^{7}$ S. Sachdev, cond-mat/0203363.

${ }^{8}$ D.P. Arovas et al., Phys. Rev. Lett. 79 (1997) 2871.

${ }^{9}$ V.F. Mitrovic et al., Nature 413 (2001) 501.

${ }^{10}$ K. Kakuyanagi et al., cond-mat/0206362.

${ }^{11}$ X.-G. Wen, P.A. Lee, Phys. Rev. Lett. 76 (1996) 503.

12 P.A. Lee, X.-G. Wen, Phys. Rev. B63 (2001) 224517.

13 J.B. Marston, I. Affleck, Phys. Rev. B39 (1989) 11538.

14 T. Hsu et al., Phys. Rev. B43 (1991) 2866.

${ }^{15}$ D.H. Kim and P.A. Lee, Annals of Phys. 272 (1999) 130.

${ }^{16}$ S. Chakravarty et al., Phys. Rev. B63 (2001) 94503.

${ }^{17}$ P.A. Lee, to be published in J. of Phys. Chem. Solids.

${ }^{18}$ D.A. Ivanov, P.A. Lee, X.-G. Wen, Phys. Rev. Lett. 84 (2000) 3958.

${ }^{19}$ P.W. Leung, Phys. Rev. B62 (2000) 6112.

${ }^{20}$ J. Kishine, P.A. Lee, X.-G. Wen, Phys. Rev. Lett. 86 (2001) 5365; Phys. Rev. B65 (2002) 064526.

${ }^{21}$ R.I. Miller et al., Phys. Rev. Lett. 88 (2002) 137002.

${ }^{22}$ Q.-H. Wang et al., Phys. Rev. Lett. 87 (2001) 167004.

${ }^{23}$ Y. Morita, J. Kishine, P.A. Lee, unpublished.

${ }^{24}$ W. Rantner, X.-G. Wen, cond-mat/0201521.

${ }^{25}$ Y. Wang et al., Phys. Rev. Lett. 88 (2002) 257003.

${ }^{26}$ P.A. Lee and N. Nagaosa, cond-mat/0211699. 